\title{
Hybrid Synchronization of a Chen Hyper-Chaotic System with Two Simple Linear Feedback Controllers
}

\author{
Guomao Xu, Shihua Chen \\ School of Mathematics and Statistics, Wuhan University, Wuhan, China \\ Email: vecent1991@whu.edu.cn
}

Received August 14, 2013; revised September 14, 2013; accepted September 21, 2013

Copyright (c) 2013 Guomao Xu, Shihua Chen. This is an open access article distributed under the Creative Commons Attribution License, which permits unrestricted use, distribution, and reproduction in any medium, provided the original work is properly cited.

\begin{abstract}
This paper brings attention on the hybrid synchronization of the Chen hyper-chaotic system by using some simple controllers. We give the sufficient conditions for achieving the goal by using the Lyapunov stability theory, and we verify our conclusion by numerical simulations.
\end{abstract}

Keywords: Hybrid Synchronization; Chen Hyper-Chaotic System; Linear Feedback Control

\section{Introduction}

Since Li and Yoke first put up the concept of chaos [1] in 1975, chaos has attracted many researchers' attention. Recently, chaos synchronization has received much more attention due to its broad application prospects in brain disorder, secure communication, and information processing, etc. [2-4]. The original synchronization technique is developed by Pecorra and Carrol in studying complete synchronization [5]. Now the concept of synchronization has been extended to a broader scope, such as anti-synchronization, lag synchronization, phase synchronization, etc. [6-9].

Although hybrid synchronization (both anti-synchronization and synchronization co-exist) has an important application in information processing [10], only a few researchers study about it. G. Li used a single variable to control the hybrid synchronization of coupled Chen system [11]. Zhang used a linear feedback control method and an adaptive feedback control method to guarantee the hybrid synchronization in general Lorenz system [12].

It is believed that the chaotic systems with higher-dimensional attractor like hyper-chaotic systems have much wider applications. In fact, the presence of more than one positive Lyapunov exponents clearly improves the security by generating more complex dynamics. Thus hyper-chaos synchronization has become a new subject of active research [13]. But there are few publications studying about it. Sudheer used active controls to accomplish the hybrid synchronization of Lü hyper-cha- otic system [13].

In this paper, we study the hybrid synchronization of a Chen hyper-chaotic system by using a simple control method. We design two linear feedback controllers so that some parts of the system are synchronized and others are anti-synchronized. We will find our method seems simpler compared to Sudheer's method [13].

\section{Problem Formulation}

We consider an autonomous chaotic system described by

$$
\dot{x}=f(x(t))
$$

where $x \in R^{n}$ is a n-dimensional state vector of the system, and $f: R^{n} \rightarrow R^{n}$ defines a vector field in $\mathrm{n}$-dimensional space, it can be a linear or nonlinear function. If $f(x(t))$ is a nonlinear function, we can also decompose it into a linear part and nonlinear part.

We often use the drive-response system method to study the synchronization of chaotic system. Thus can be described by

$$
\dot{s}_{1}(t)=f\left(s_{1}(t)\right)
$$

and:

$$
\dot{s}_{2}(t)=f\left(s_{2}(t)\right)+u\left(s_{1}(t), s_{2}(t)\right)
$$

where $u\left(s_{1}(t), s_{2}(t)\right)$ is a controller to be designed which can be linear, linear or other form. (2) is called the drive system and (3) is called the response system.

Definition 1. The chaotic system (2) and (3) are called 
to achieve hybrid synchronization, if the following situations are satisfied:

$$
\begin{aligned}
& \lim _{t \rightarrow+\infty}\left|s_{1 i}(t)+s_{2 i}(t)\right|=0, i=1,2, \cdots, m, \\
& \lim _{t \rightarrow+\infty}\left\|s_{1 i}(t)-s_{2 i}(t)\right\|=0, i=m+1, \cdots, n .
\end{aligned}
$$

where $s_{1 i}(t), s_{2 i}(t)$ are the component of the state vectors. If only (4) is satisfied, then we call the two system -achieve anti-synchronization. If only (5) is satisfied, then we call the two system achieves synchronization.

\section{Main Result}

The differential equations of Chen hyper-chaotic system [14] is described by:

$$
\begin{aligned}
& \dot{x}=a(y-x)+w \\
& \dot{y}=d x-x z+c y \\
& \dot{z}=x y-b z \\
& \dot{w}=y z+r w
\end{aligned}
$$

where $x, y, z$, and $w$ are state variables, and $a, b, c, d, r$ are positive parameters. When $a=35, b=3, c=12$, $d=7$. $0 \leq r \leq 0.085$, system (6) is chaotic, when $a=35, b=3, c=12, d=7.0 .085<r \leq 0.798$, system (6) is hyper-chaotic, when $a=35, b=3, c=12$, $d=7.0 .798<r \leq 0.9$, system (6) is periodic.

In order to observe the hybrid synchronization behavior of Chen hyper-chaotic system, assume that we have two Chen hyper-chaotic systems where the drive system with four state variables denoted by the subscript 1 and the response system with identical equations denoted by the subscript 2. Obviously, the initial condition on the drive system is different from that of response system. For the system (6), the drive and response systems are defined below, respectively:

$$
\begin{aligned}
& \dot{x}_{1}=a\left(y_{1}-x_{1}\right)+w_{1} \\
& \dot{y}_{1}=d x_{1}-x_{1} z_{1}+c y_{1} \\
& \dot{z}=x_{1} y_{1}-b z_{1} \\
& \dot{w}_{1}=y_{1} z_{1}+r w_{1}
\end{aligned}
$$

and

$$
\begin{aligned}
& \dot{x}_{2}=a\left(y_{2}-x_{2}\right)+w_{2} \\
& \dot{y}_{2}=d x_{2}-x_{2} z_{2}+c y_{2} \\
& \dot{z}_{2}=x_{2} y_{2}-b z_{2} \\
& \dot{w}_{2}=y_{2} z_{2}+r w_{2}
\end{aligned}
$$

where $u_{1}, u_{2}, u_{3}, u_{4}$ are four control functions to be designed. In order to study the hybrid synchronization of the two systems (7) and (8), we defined an error variable as

$$
e=\left(e_{1}, e_{2}, e_{3}, e_{4}\right)^{\mathrm{T}}=\left(x_{1}+x_{2}, y_{1}+y_{2}, z_{1}-z_{2}, w_{1}+w_{2}\right)^{\mathrm{T}} .
$$

Lemma 1. (Schur's formula) Let $P$ be a square matrix partitioned as [15]

$$
\left(\begin{array}{ll}
A & B \\
C & D
\end{array}\right) .
$$

If $A$ is nonsingular, then:

$$
\operatorname{det}(P / A)=\operatorname{det}(P) / \operatorname{det}(A),
$$

where

$$
(P / A)=D-C A^{-1} B .
$$

Then we will discuss the hybrid synchronization of a Chen hyper-chaotic system below.

Based on the error vector $e$, we can derive the error dynamical system:

$$
\begin{aligned}
& \dot{e}_{1}=a\left(e_{2}-e_{1}\right)+e_{4}+u_{1} \\
& \dot{e}_{2}=d e_{1}+c e_{2}-x_{2} z_{2}-x_{1} z_{1}+u_{2} \\
& \dot{e}_{3}=-b e_{3}-x_{2} y_{2}+x_{1} y_{1}-u_{3} \\
& e_{4}=r e_{4}+y_{2} z_{2}+y_{1} z_{1}+u_{4}
\end{aligned}
$$

Obviously, from the viewpoint of control theory, if the error vector $e$ converge to zero as time $t$ goes to infinity, i.e. asymptotical stability. As system (6) is hyper-chaotic, thus $y$ and $z$ are all bounded. We suppose that $N$ and $M$ are the upper bounds of the absolute values of $y$ and $z$. Then we can conclude that hybrid synchronization exists between system (7) and (8).

Theorem 1. The hybrid synchronization existent in system (7) and (8) if we choose $u_{1}=0, u_{2}=-k_{1} e_{2}$, $u_{3}=0, u_{4}=-k_{2} e_{4}$ as the control functions, where $k_{1}>\frac{(a p+d+M)^{2} b}{4 a p b-N^{2}}+c$ and $k_{2}$ satisfies $k_{2}>r$ and $\operatorname{det}\left(C-B^{\mathrm{T}} * A^{-1} * B\right)>0$ where

$$
A=\left(\begin{array}{cc}
a p & \frac{N}{2} \\
-\frac{N}{2} & b
\end{array}\right)>0, B=\left(\begin{array}{cc}
-\frac{a p+d+M}{2} & -\frac{p}{2} \\
0 & -\frac{N}{2}
\end{array}\right) \text {, }
$$

$$
C=\left(\begin{array}{cc}
k_{1}-c & -\frac{M}{2} \\
-\frac{M}{2} & k_{2}-r
\end{array}\right) .
$$

Proof: The control function $U=\left[u_{1}, u_{2}, u_{3}, u_{4}\right]^{\mathrm{T}}$

$$
\begin{aligned}
& u_{1}=0 \\
& u_{2}=-k_{1} e_{1} \\
& u_{3}=0 \\
& u_{4}=-k_{2} e_{2}
\end{aligned}
$$

This leads to 


$$
\begin{aligned}
& \dot{e}_{1}=a\left(e_{2}-e_{1}\right)+e_{4} \\
& \dot{e}_{2}=d e_{1}+c e_{2}-x_{2} z_{2}-x_{1} z_{1}-k_{1} e_{2} \\
& \dot{e}_{3}=-b e_{3}-x_{2} y_{2}+x_{1} y_{1} \\
& e_{4}=r e_{4}+y_{2} z_{2}+y_{1} z_{1}-k_{2} e_{4}
\end{aligned}
$$

Now we define the Lyapunov function for the system (11) as

$$
V(t)=\frac{1}{2}\left(p e_{1}^{2}+e_{2}^{2}+e_{3}^{2}+e_{4}^{2}\right)
$$

Its time derivative along the trajectories system (11) is

$$
\begin{aligned}
& \dot{V}(t)=p e_{1} \dot{e}_{1}+e_{2} \dot{e}_{2}+e_{3} \dot{e}_{3}+e_{4} \dot{e}_{4} \\
& =p e_{1}\left[a\left(e_{2}-e_{1}\right)+e_{4}\right]+e_{2}\left[d e_{1}+\left(c-k_{1}\right) e_{2}-e_{1} z_{1}+x_{2} e_{3}\right] \\
& +e_{3}\left(e_{1} y_{1}-x_{2} e_{2}-b e_{3}\right)+e_{4}\left[e_{2} z_{1}-y_{2} e_{3}+\left(r-k_{4}\right) e_{4}\right] \\
& =-p a e_{1}^{2}+\left(c-k_{1}\right) e_{2}^{2}-b e_{3}^{2}+\left(r-k_{2}\right) e_{4}^{2}+y_{1} e_{1} e_{3} \\
& +\left(a p+d-z_{1}\right) e_{1} e_{2}+p e_{1} e_{4}+z_{1} e_{2} e_{4}-y_{2} e_{3} e_{4} \\
& \leq-p a e_{1}^{2}+\left(c-k_{1}\right) e_{2}^{2}-b e_{3}^{2}+\left(r-k_{2}\right) e_{4}^{2}+M\left|e_{1} e_{3}\right| \\
& +(a p+d+M)\left|e_{1} e_{2}\right|+p\left|e_{1} e_{4}\right|+M\left|e_{2} e_{4}\right|+N\left|e_{3} e_{4}\right| \\
& =\left(\left|e_{1}\right|,\left|e_{3}\right|,\left|e_{2}\right|,\left|e_{4}\right|\right) Q\left(\left|e_{1}\right|,\left|e_{3}\right|,\left|e_{2}\right|,\left|e_{4}\right|\right)^{\mathrm{T}}
\end{aligned}
$$

where

$$
Q=\left(\begin{array}{cc}
A & B \\
B^{\mathrm{T}} & C
\end{array}\right)
$$

Obviously, from the conditions of the theorem we have $a p>0$, $\operatorname{det} Q_{22}>0$, det $Q_{33}>0$, and base on Lemma1, we know that $\operatorname{det}(Q)>0 . Q_{22}, Q_{33}$ denote the 2th and 3th order principal minor of $Q$, that is, $Q$ is positive definite. Thus the error system (9) is asymptotical stability at $e=0$. Thus the theorem is proved.

We can easily find out that Sudheer used some complex controllers to achieve their goal, and it seems not so useful in practical application.

\section{Numerical Simulations}

In the numerical simulations, we use the fourth-order Runge-Kutta method to solve the systems. Assume that the initial conditions of the drive and response system are $\left(x_{1}(0), y_{1}(0), z_{1}(0), w_{1}(0)\right)=(0.1,0.5,2,0.8)$, $\left(x_{2}(0), y_{2}(0), z_{2}(0), w_{2}(0)\right)=(1,2.5,5,0.2)$. Hence the initial values of error system are

$\left(e_{1}(0), e_{2}(0), e_{3}(0), e_{4}(0)\right)=(1.1,3,-3,1)$. We choose $a=35, b=3, c=12, d=7, r=0.5$, thus through theorem 1 we can take $k_{1}=250, k_{2}=250, p=5$, $M=40, N=30$. Thus

$$
A=\left(\begin{array}{cc}
175 & -15 \\
-15 & 3
\end{array}\right)>0 \quad B=\left(\begin{array}{cc}
-111 & -2.5 \\
0 & 15
\end{array}\right)
$$

$$
C=\left(\begin{array}{cc}
238 & -20 \\
-20 & -249.5
\end{array}\right)
$$

Thus it's easy to verify that these matrixes are satisfied the conditions. And $Q=\left(\begin{array}{cc}A & B \\ B^{\mathrm{T}} & C\end{array}\right)>0$.

From Figures 1-4, we can easily see that $e_{1}=x_{1}+x_{2} \rightarrow 0, e_{2}=y_{1}+y_{2} \rightarrow 0, e_{3}=z_{1}-z_{2} \rightarrow 0$, $e_{4}=w_{1}+w_{2} \rightarrow 0$ as time $t$ tends to infinite.

\section{Conclusion}

In this paper, we study the problem of chaos hybrid synchronization of Chen hyper-chaotic system, i.e., some parts of states are anti-synchronization; other parts of states are synchronization. We can use active control theory to synchronize and anti-synchronize hyper-chaotic systems. Numerical simulations are used to verify the effectiveness of the proposed control method.

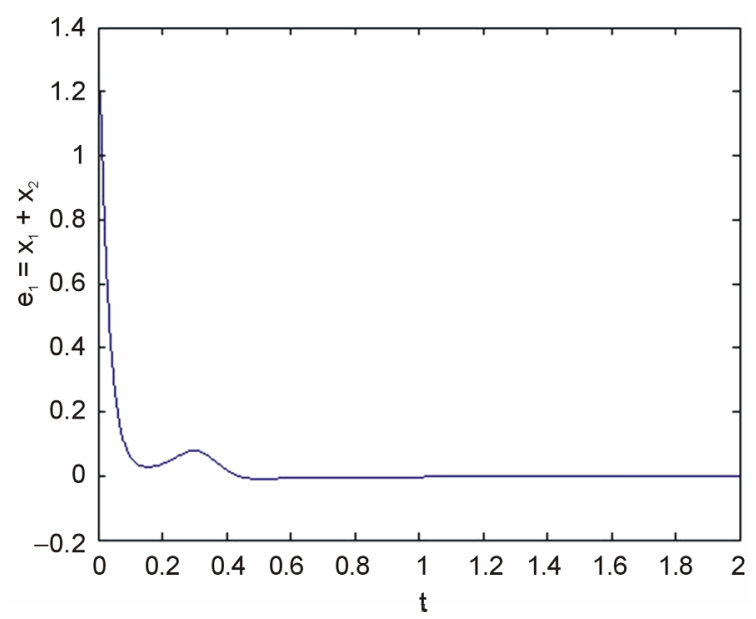

(a)

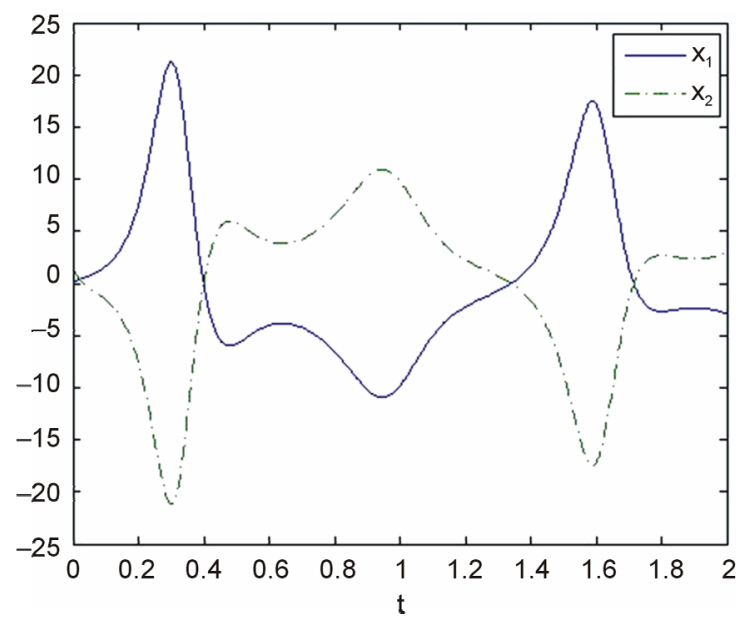

(b)

Figure 1. (a) Shows the behavior of trajectory $e_{1}$; (b) Shows the behavior of the trajectory $x_{1}$ and $x_{2}$. 


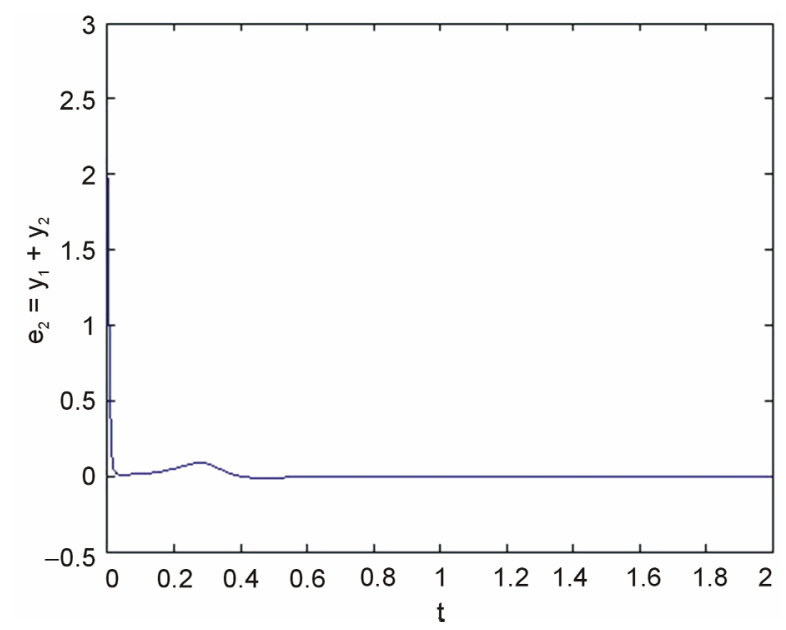

(a)

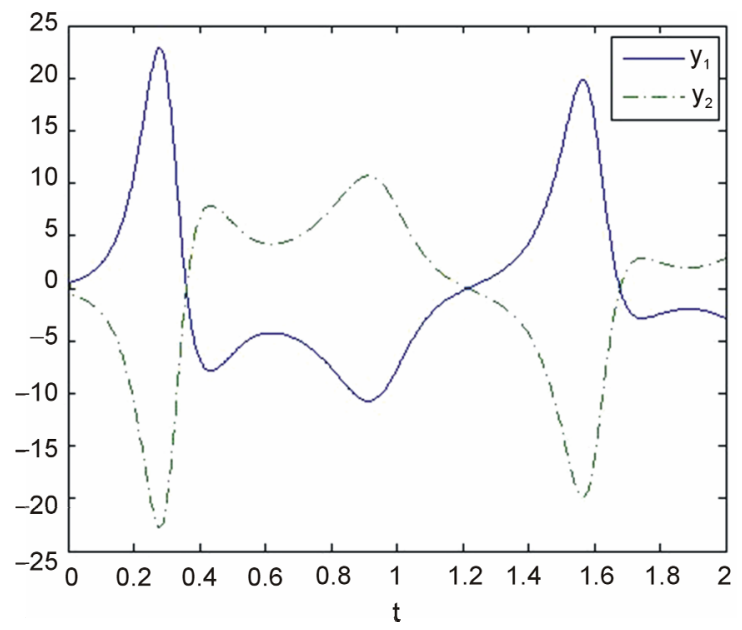

(b)

Figure 2. (a) Shows the behavior of trajectory $e_{2}$; (b) Shows the behavior of the trajectory $y_{1}$ and $y_{2}$.

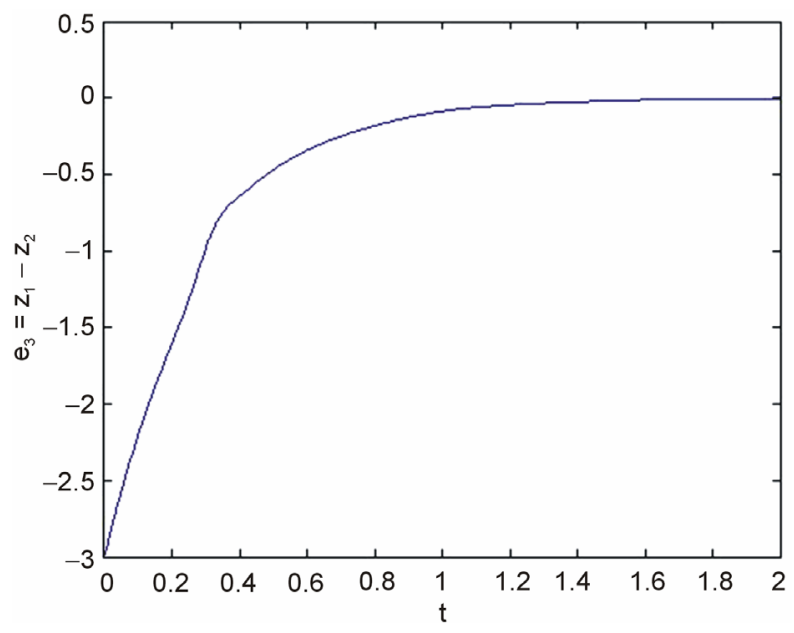

(a)

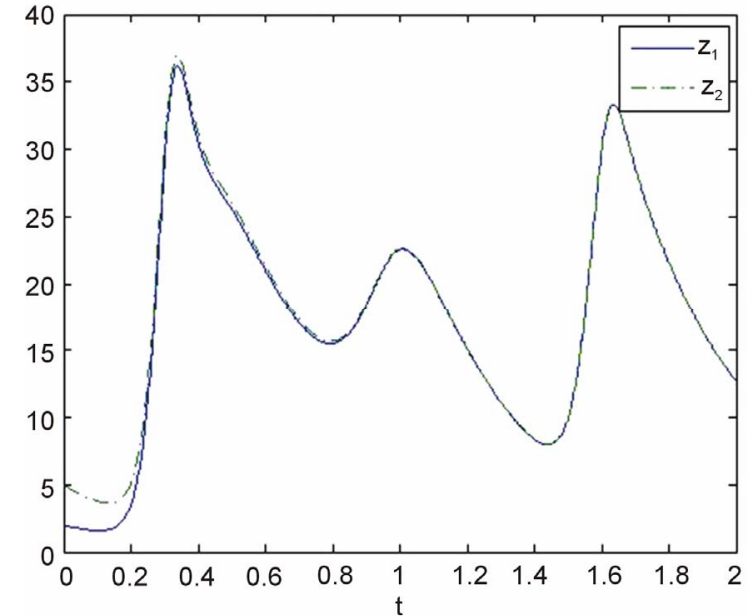

(b)

Figure 3. (a) Shows the behavior of trajectory $e_{3}$; (b) Shows the behavior of the trajectory $z_{1}$ and $z_{2}$.

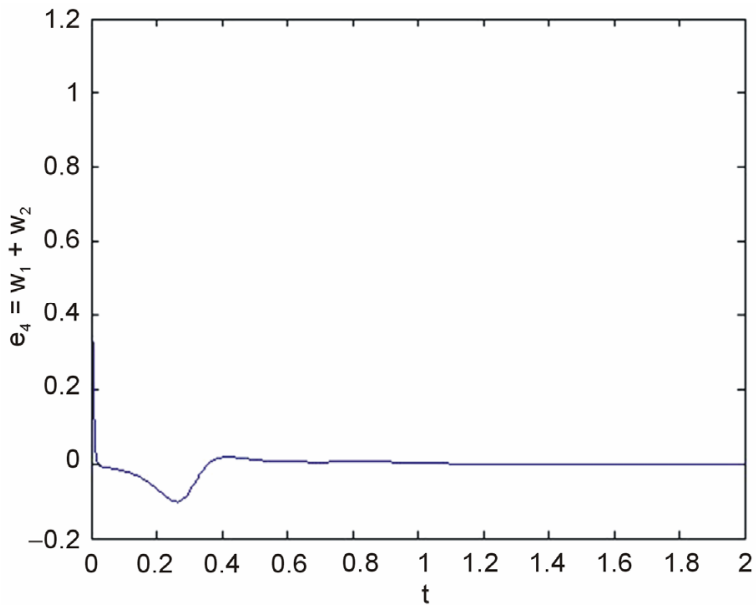

(a)

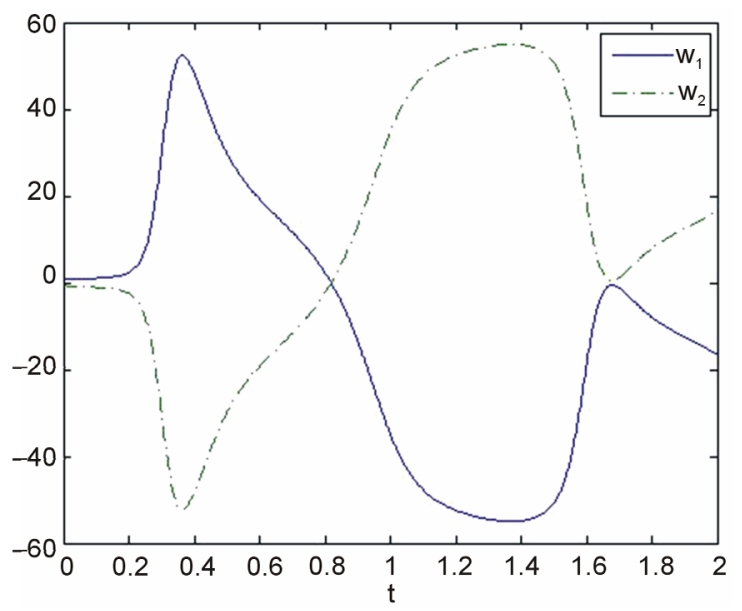

(b)

Figure 4. (a) Shows the behavior of trajectory $e_{4}$; (b) Shows the behavior of the trajectory $w_{1}$ and $w_{2}$. 


\section{Acknowledgements}

This work is supported by the National Natural Science Foundation (NNSF) of China under Grant No. 61273215.

\section{REFERENCES}

[1] T. Y. Li and J. A. Yorke, "Period Three Implies Chaos," The American Mathematical Monthly, Vol. 82, No. 10. 1975, pp. 985-992. http://dx.doi.org/10.2307/2318254

[2] E. Rodriguez, N. George, J. P. Lachaux, J. Martinerie, B. Renault and F. J. Varela, "Perception's Shadow: LongDistance Synchronization of Human Brain Activity," Nature, Vol. 397, No. 6718, 1999, pp. 430-433.

[3] R. Yoshida, M. Tanaka, S. Onodera, T. Yamaguchi and E. Kokufuta, "In-Phase Synchronization of Chemical and Mechanical Oscillations in Self-Oscillating Gels," The Journal of Physical Chemistry A, Vol. 104, No. 32, 2000, pp. 7549-7555. http://dx.doi.org/10.1021/jp0011600

[4] W. Singer, "Synchronization of Cortical Activity and Its Putative Role in Information Processing and Learning," Annual Review of Physiology, Vol. 55, No. 1, 1993, pp. 349-374.

http://dx.doi.org/10.1146/annurev.ph.55.030193.002025

[5] L. M. Pecora and T. L. Carroll, "Synchronization in Chaotic Systems,” Physical Review Letters, Vol. 64, No. 8, 1990, pp. 821-824. http://dx.doi.org/10.1103/PhysRevLett.64.821

[6] S. Boccaletti, J. Kurths, G. Osipov, D. L. Valladares and C. S. Zhou, "The Synchronization of Chaotic Systems," Physics Reports, Vol. 366, No. 1, 2002, pp. 1-101. http://dx.doi.org/10.1016/S0370-1573(02)00137-0

[7] M. G. Rosenblum, A. S. Pikovsky and J. Kurths, "Phase Synchronization of Chaotic Oscillators," Physical Review Letters, Vol. 76, No. 11, 1996, pp. 1804-1807. http://dx.doi.org/10.1103/PhysRevLett.76.1804
[8] R. Mainieri and J. Rehacek, "Projective Synchronization in Three-Dimensional Chaotic Systems," Physical Review Letters, Vol. 82, No. 15, 1999, pp. 3042-3045. http://dx.doi.org/10.1103/PhysRevLett.82.3042

[9] C. M. Kim, S. Rim, W. H. Kye, J. W. Ryu and Y. J. Park, "Anti-Synchronization of Chaotic Oscillators," Physics Letters A, Vol. 320, No. 1, 2003, pp. 39-46. http://dx.doi.org/10.1016/j.physleta.2003.10.051

[10] Q. Xie, G. Chen and E. M. Bollt, "Hybrid Chaos Synchro Nization and Its Application in Information Processing," Mathematical and Computer Modeling, Vol. 35, No. 1, 2002, pp. 145-163. http://dx.doi.org/10.1016/S0895-7177(01)00157-1

[11] C. Li, Q. Chen and T. Huang, “Coexistence of Anti-Phase and Complete Synchronization in Coupled Chen System via a Single Variable,” Chaos, Solitons \& Fractals, Vol. 38, No. 2, 2008, pp. 461-464. http://dx.doi.org/10.1016/j.chaos.2006.11.028

[12] Q. Zhang, J. Lü and S .Chen, “Coexistence of Anti-Phase and Complete Synchronization in the Generalized Lorenz System," Communications in Nonlinear Science and $\mathrm{Nu}$ merical Simulation, Vol. 15, No. 10, 2010, pp. 30673072. http://dx.doi.org/10.1016/j.cnsns.2009.11.020

[13] K. S. Sudheer and M. Sabir, "Hybrid Synchronization of Hyperchaotic Lu System,” Pramana, Vol. 73, No. 4, 2009, pp. 781-786. http://dx.doi.org/10.1007/s12043-009-0145-1

[14] Y. Li, W. K. Tang and G. Chen, "Generating Hyperchaos via State Feedback Control,” International Journal of Bifurcation and Chaos, Vol. 15, No. 10, 2005, pp. 33673375.

[15] F. Zhang, "The Schur Complement and Its Applications," Springer Science \& Business Media, Inc., Boston, 2005. http://dx.doi.org/10.1007/b105056 